\title{
A NOTE ON THE SINGULAR POINTS OF THE FUNCTION GENERATED BY THE BERGMAN OPERATOR OF THE SECOND KIND
}

\author{
PAUL ROSENTHAL
}

\begin{abstract}
Let $\psi=P_{2}(f)$ be Bergman's operator of the second kind, $f(q)$ is analytic at $q=0$. In a previous paper [5] a theorem was obtained on the singularities of $\psi$ when $\psi$ was generated by a $f(q)$ whose only singular points were poles. In this note we obtain a theorem on the singularities of $\psi$ when $\psi$ is generated by a $f(q)$ whose singular points can be of more varied types.
\end{abstract}

Introduction. The method of integral operators developed by Bergman in [2] associates an operator solution $\psi=P_{2}(f)$ to the partial differential equation of mixed type $\psi_{x x}+l(x) \psi_{y y}=0, l(x)>0, x<0, l(x)<0, x>0$. The use of such an operator enables one to solve certain types of boundary value problems $[2$, p. 120] which arise in physical applications. Since $P_{2}(f)$ maps analytic functions $f(q)$ into solutions of the above equation, the problem of the behavior of the solution $\psi(x, y)$ when $f(q)$ has singularities of specified types is solved in this paper. This appears to be of interest in some problems of mathematical physics. We consider the Tricomi case only. In this case $\psi=P_{2}(f)$ maps functions $f(q)$ analytic at $q=0$ into solutions of the partial differential equation

$$
\psi_{z z^{*}}+4 F \psi=0, \quad F=(5 / 36)\left(z+z^{*}\right)^{-2} \quad \text { (Tricomi case). }
$$

For this case a solution to (1) can be written as

$$
\begin{aligned}
& \psi\left(z, z^{*}\right)=\int_{-1}^{1}( A_{1} F\left(\frac{1}{6}, \frac{5}{6}, \frac{1}{2}, \frac{t^{2} z}{z+z^{*}}\right) \\
&\left.+A_{2}\left(\frac{z}{z+z^{*}}\right)^{1 / 2} t F\left(\frac{2}{3}, \frac{4}{3}, \frac{3}{2}, \frac{t^{2} z}{z+z^{*}}\right)\right) \\
& \\
& \times f\left(\frac{1}{2} z\left(1-t^{2}\right)\right) \frac{d t}{\left(1-t^{2}\right)^{1 / 2}},
\end{aligned}
$$

Received by the editors March 5, 1973 and, in revised form, August 30, 1973.

AMS (MOS) subject classifications (1970). Primary 35C15, 35J15; Secondary 30A10, 30A68, 33A30, 44A15.

Key words and phrases. Hypergeometric function, singular points. 
$F \equiv$ hypergeometric function, $z, z^{*}$ complex variables, $A_{1}, A_{2}$ complex constants, $-1<t<1$ (all our integrals are in the improper Riemann sense (complex)), see [1, p. 871], [2, pp. 107, 108].

One can also obtain a second solution to (1),

$$
\psi_{0}\left(z, z^{*}\right)=\int_{-1}^{1}\left(B_{1} u^{-1 / 6} F\left(\frac{1}{6}, \frac{2}{3}, \frac{1}{3}, \frac{1}{u}\right)+B_{2} u^{-5 / 6} F\left(\frac{5}{6}, \frac{4}{3}, \frac{5}{3}, \frac{1}{u}\right)\right)
$$

$$
\begin{aligned}
\times f\left(\frac{1}{2} z\left(1-t^{2}\right)\right) & \frac{d t}{\left(1-t^{2}\right)^{1 / 2}}, \\
u & =\frac{t^{2} z}{z+z^{*}} ;
\end{aligned}
$$

see $\left[1\right.$, p. 871]. We note $\psi_{0}\left(z, z^{*}\right)$ of (3) is the analytic continuation of $\psi\left(z, z^{*}\right)$ of (2) for a suitable choice of the path of integration and constants $B_{1}, B_{2}$, see [1, p. 873].

THEOREM. Let $f(q)=\sum_{n=0}^{\infty} a_{n}(q \mid A)^{n}, \operatorname{Re}, \operatorname{Im} A>0$, where

(a) $\lim \sup _{n \rightarrow \infty}\left|a_{n}\right|^{1 / n}=1,\left|\arg a_{n}\right|<\varepsilon, 0<\varepsilon<\pi / 2, n$ sufficiently large, or

(b) $\lim _{n \rightarrow \infty} a_{n+1} / a_{n}=1$. Then $\psi\left(z, z^{*}\right)$ of (2) is singular at the points $z=2 A, z^{*}=2 \alpha A, 1 \geqq \alpha>0$.

Proof. Let $z^{*}=\alpha z, 1 \geqq \alpha>0, z \neq 0$. We note the first integral in (2) is an even function of $t$, the second an odd function of $t$. When $|z| \leqq|2 A|-$ $\delta,|2 A|>\delta>0$, we are permitted to interchange the order of integration and summation in (2). Thus we obtain from (2)

$$
g_{\alpha}(z)=\psi(z, \alpha z)=2 A_{1} \sum_{n=0}^{\infty} \alpha_{n}(\alpha) a_{n}\left(\frac{z}{2 A}\right)^{n}, \quad|z|<|2 A|,
$$

where

$$
\begin{aligned}
& \alpha_{n}(\alpha)=\int_{0}^{1} F\left(\frac{1}{6}, \frac{5}{6}, \frac{1}{2}, \frac{t^{2}}{1+\alpha}\right)\left(1-t^{2}\right)^{n-1 / 2} d t, \\
& n \geqq 0, \quad 1 \geqq \alpha>0 .
\end{aligned}
$$

Since $F$ is monotonic in $t$, i.e., if $1 \geqq t_{1}>t_{2} \geqq 0$, then $F\left(t_{1}\right)>F\left(t_{2}\right)$, we infer the following bounds for $\alpha_{n}(\alpha)$,

$$
\begin{array}{r}
h(\alpha) \geqq \alpha_{n}(\alpha) \geqq 2^{-1}\left(\Gamma\left(\frac{1}{2}\right) \Gamma\left(n+\frac{1}{2}\right)\right) / \Gamma(n+1)>0, \\
n \geqq 0, \Gamma \equiv \text { Gamma function, }
\end{array}
$$

$h(\alpha)$ is independent of $n$, the right-hand bound in (6a) is obtained by letting $t=0$ in $F(t)$ and thus obtaining the Beta integral $\int_{0}^{1}(1-s)^{n-1 / 2} s^{-1 / 2} d s$ 
from (5). We also obtain from (5)

$$
\begin{gathered}
\alpha_{n}(\alpha)=\sum_{s=0}^{\infty} 2^{-1} f_{1}(\alpha, s, n), \quad f_{1}(\alpha, s, n)=f_{2}(\alpha, s) \frac{\Gamma\left(s+\frac{1}{2}\right) \Gamma\left(n+\frac{1}{2}\right)}{\Gamma(s+n+1)}, \\
f_{2}(\alpha, s)=\frac{\Gamma\left(\frac{1}{2}\right)}{\Gamma\left(\frac{1}{6}\right) \Gamma\left(\begin{array}{c}
5 \\
6
\end{array}\right)} \frac{\left.\Gamma\left(\frac{1}{6}+s\right) \Gamma\left(\begin{array}{l}
5 \\
6
\end{array}\right)+s\right)}{\Gamma\left(\frac{1}{2}+s\right) \Gamma(1+s)} \frac{1}{(1+\alpha)^{s}} .
\end{gathered}
$$

(6b) is obtained from (5) by using the series definition for $F$ and then interchanging summation and integration which is permissible since we have dominated convergence for $1 \geqq \alpha>0$.

Using next the hypothesis of our Theorem, (6a), (6b), we obtain

$$
\begin{aligned}
\limsup _{n \rightarrow \infty}\left|\alpha_{n}(\alpha) a_{n}\right|^{1 / n}=\lim _{n \rightarrow \infty}\left|\alpha_{n}(\alpha)\right|^{1 / n} & \limsup _{n \rightarrow \infty}\left|a_{n}\right|^{1 / n}=1, \\
& \left|\arg \left(\alpha_{n}(\alpha) a_{n}\right)\right|<\varepsilon, 0<\varepsilon<\pi / 2,
\end{aligned}
$$

$n$ sufficiently large. Since $\left(\mathrm{a}^{*}\right)$ satisfies the conditions of a theorem of Dienes, see [4, p. 227], we conclude $z=2 A$ is a singular point of $g_{\alpha}(z)$ of (4). Or

$$
\lim _{n \rightarrow \infty}\left(\alpha_{n+1}(\alpha) / \alpha_{n}(\alpha)\right)\left(a_{n+1} / a_{n}\right)=1 ;
$$

the conclusion $\left(\mathrm{b}^{*}\right)$ follows from the result $\lim _{n \rightarrow \infty} \alpha_{n+1}(\alpha) / \alpha_{n}(\alpha)=1$ which follows from (6b) when we form the ratio $\alpha_{n+1} / \alpha_{n}$ and then noting we can interchange the limit operation and summation since we have dominated convergence for large $n$. Since $\left(\mathrm{b}^{*}\right)$ satisfies the condition of a theorem of Fabry, see [3, p. 307], we conclude $z=2 A$ is a singular point of $g_{\alpha}(z)$ of (4), $1 \geqq \alpha>0$. Assume $\psi\left(z, z^{*}\right)$ of (2) admits the Taylor expansion

$$
\begin{aligned}
\psi\left(z, z^{*}\right)=\sum_{m, n=0}^{\infty} a_{m n}(z-2 A)^{m}\left(z^{*}-2 \alpha A\right)^{n}, \\
\quad|z-2 A|<\delta_{1},\left|z^{*}-2 \alpha A\right|<\delta_{2}, \delta_{1}, \delta_{2}>0 .
\end{aligned}
$$

Using the conditions $1 \geqq \alpha>0, \delta^{*}=\min \left(\delta_{1}, \delta_{2}\right), z^{*}=\alpha z$, we conclude when $|z-2 A|<\delta^{*}$, then $\left|z^{*}-2 \alpha A\right|<\delta^{*}$. Since we have absolute convergence of the double series in (7) for $|z-2 A|<\delta^{*}$, we are permitted to rearrange the right-hand side of (7) to conclude

$$
g_{\alpha}(z)=\sum_{n=0}^{\infty} b_{n}(\alpha)(z-2 A)^{n}, \quad|z-2 A|<\delta^{*}, \delta^{*}>0,
$$

where $b_{0}=a_{00}, b_{1}(\alpha)=\left(a_{01} \alpha+a_{10}\right), b_{2}(\alpha)=\left(a_{02} \alpha^{2}+a_{11} \alpha+a_{20}\right), \cdots, 1 \geqq \alpha>0$. Hence we conclude $g_{\alpha}(z)$ of (4) is analytic at $z=2 A$ which contradicts $\left(a^{*}\right)$ or $\left(b^{*}\right)$ of above. This completes the proof of our Theorem. 
We note in closing, there exist nonempty neighborhoods $N_{\delta}(z), \operatorname{Re}$, $\operatorname{Im} z>0, N_{\delta}\left(z^{*}\right), \operatorname{Re}, \operatorname{Im} z^{*}>0$, satisfying the inequalities $|z|<\left|z+z^{*}\right|$, $|z|<|2 A|$, see (2) and (4). Hence $\psi\left(z, z^{*}\right)$ of (2) is analytic in $z$ and $z^{*}$ for $z \in N_{\delta}(z), z^{*} \in N_{\delta}\left(z^{*}\right)$.

\section{REFERENCES}

1. S. Bergman, Two-dimensional transonic flow patterns, Amer. J. Math. 70 (1948), 856-891. MR 10, 752.

2. - Integral operators in the theory of linear partial differential equations, 2nd rev. ed., Ergebnisse der Math. und ihrer Grenzgebiete, Band 23, Springer-Verlag, New York, 1969. MR 39 \#596.

3. L. Bierberbach, Lehrbuch der Funktionstheorie. II, Teubner, Leipzig, 1931.

4. P. Dienes, The Taylor series, Oxford Univ. Press, 1931.

5. P. Rosenthal, On the location of the singularities of the function generated by the Bergman operator of the second kind, Proc. Amer. Math. Soc. 44 (1974), 157-162.

Department of Mathematics, Stanford University, Stanford, California 94305

Current address: Apt. 103, 90 Heather Avenue, San Francisco, California 94118 